\title{
Principios de clasificación y enmarcación en la evaluación de la lengua materna
} Principles of classification and framing in mother tongue evaluation

Recibido: 4 de febrero de 2013 / Aprobado: 3 de mayo de 2013

\author{
Elssa Ibeth Valbuena Valbuena* \\ Nidia Omaira Valbuena Valbuena*
}

\section{Resumen}

Este artículo analiza los resultados de la investigación sobre la evaluación de los aprendizajes a la luz de los principios de clasificación y enmarcación, con el ánimo de establecer la relación entre procesos de comprensión y cualificación de las prácticas evaluativas desde una perspectiva crítica. El diseño metodológico corresponde al estudio de caso y sus unidades analíticas son los discursos y las prácticas evaluativas de cuatro profesores de lengua castellana. Se utilizaron instrumentos como grabaciones de audio y video, observaciones no participantes de clase y registros escritos, entre otros. La investigación permitió determinar que en el ámbito evaluativo del caso estudiado prevalece el discurso regulativo sobre el instruccional.

Palabras clave: discursos, prácticas evaluativas, evaluación formativa, clasificación, enmarcación.

\section{Abstract}

The paper presents research results "Discourses and evaluative practices in the language classification and framing. The main objective is establishing the relations between knowledge, power and forms of consciousness that are present in the language classroom. It aims to promote the methodological design corresponds to the case study and its analytical units are speeches recordings, class observations, among others. The investigation established that in evaluation prevails the regulative discourse.

Keywords: formative evaluation, discourses, evaluative practices, classification, framing.

\section{Introducción}

El problema de la evaluación de los aprendizajes en el aula constituye el objeto de reflexión y cualificación de la investigación cuya síntesis se presenta en este escrito. Este tema es una de las preocupaciones de la investigación social contemporánea por cuanto tiene multiplicidad de dimensiones y como tal, re-

sulta ser un proceso complejo que ha de ubicarse en el plano pedagógico, ya que trasciende la vida de los sujetos implicados en este.

El artículo presenta, en primer lugar, algunas consideraciones relacionadas con los antecedentes investigativos sobre la evaluación de los aprendiza-

\footnotetext{
* Licenciada en lingüística y literatura. Especialista en lenguaje y pedagogía de proyectos. Egresada de Maestría en Pedagogía de la Lengua Materna de la Universidad Distrital Francisco José de Caldas. Docente de Lengua Castellana de la Institución Educativa Departamental Serrezuela. Integrante del grupo de investigación Lenguaje, Sentido y Contexto. Correo electrónico: ibethval@yahoo.com.

** Licenciada en lingüística y literatura. Especialista en lenguaje y pedagogía de proyectos. Egresada de la Maestría en Pedagogía de la Lengua Materna de la Universidad Distrital Francisco José de Caldas. Docente de Lengua Castellana en la Universidad del Tolima. Integrante del grupo de investigación Lenguaje, Sentido y Contexto. Correo electrónico: nvalbuenita@yahoo.com.
} 
jes. Primero, se exponen los elementos conceptuales que orientaron la investigación, entre los cuales se destacan planteamientos teóricos que abordan la evaluación de los aprendizajes de lengua materna desde una perspectiva crítica: la evaluación formativa, el discurso instruccional y las consignas para la realización de tareas. Se dan a conocer los principios de clasificación y enmarcación propuestos por Basil Bernstein, por cuanto constituyen la base teórica para el análisis de datos.

En segundo lugar se presenta la metodología que orientó la investigación. El enfoque, las fases, las estrategias y los instrumentos, las categorías y procedimientos analíticos, el diseño de la investigación y sus etapas, las características de la población objeto de estudio, los instrumentos y las estrategias aplicadas en la recolección y el análisis de datos. El análisis de los discursos evaluativos se hace mediante las consignas para establecer la función que cumplen en la evaluación y en la enseñanza de lengua y literatura. También se analiza el discurso pedagógico, el cual está constituido por el discurso instruccional y el regulativo. El primero se relaciona directamente con el principio de clasificación y el segundo con el principio de enmarcación. Después se analizan los efectos performativos ${ }^{1}$ que causan estos principios en los estudiantes y se discuten los resultados. Los datos permiten inferir que los discursos de formación docente se recontextualizan en aula; existe una débil formación docente y fuertes exigencias de las agencias educativas. Finalmente se presentan las conclusiones.

\section{Antecedentes investigativos}

Las tendencias investigativas actuales sobre la evaluación de los aprendizajes en lengua materna evidencian distintos enfoques. Unos trabajos investigativos se orientan a estudiar la evaluación externa, otros a la evaluación interna y otros centran su atención al estudio de la evaluación desde una perspectiva crítica.

Dentro de las investigaciones cuyo interés se centra en la evaluación externa se destacan, por una parte, los trabajos de Jurado (2003, 2005, 2009), Atorresi (2006, 2009) y Pérez (2007), quienes mediante los resultados de pruebas externas nacionales e internacionales, intentan orientar procesos evaluativos que permitan superar el déficit de los estudiantes en las pruebas y, al mismo tiempo, buscan orientar el diseño de políticas educativas nacionales y el desarrollo de procesos de formación docente. Por otra parte, se encuentran los trabajos desarrollados por Rodríguez (2010) y Camargo Gutiérrez y Pedraza (2006), los cuales intentan develar las concepciones de los profesores sobre la evaluación de la producción escrita. Los resultados plantean que hay un evidente predominio de concepciones tradicionales sobre la evaluación de la escritura. Los trabajos de Camargo, Uribe y Caro (2007) y Castillón, Camargo y Caro (2010) analizan una muestra de los relatos presentados al Concurso Nacional de Cuento Gabriel García Márquez en 2007, para hacer hipótesis frente a los procesos de enseñanza y aprendizaje de la escritura y a partir de los resultados proponer programas de formación docente.

La revisión de las investigaciones relacionadas con la evaluación interna permitió establecer que hay dos perspectivas teóricas, no excluyentes, que orientan el estudio del proceso de la producción textual: una social, con influencia de la dimensión pragmática del lenguaje y otra cognitiva. Entre las investigaciones cuya perspectiva teórica se ubica en el aspecto pragmático de la producción textual se encuentran las desarrolladas por Camelo (2010), Herrera et al. (2008), Saavedra (2006). Las investigaciones que priorizan en los aspectos cognitivos involucrados

1 Desde la teoría de los actos de habla propuesta por Austin (1962) los efectos performativos no son utilizados para describir o constatar determinadas cosas, sino que se interesan por la realización de un acto. Es la conducta que causa el enunciado en los interlocutores. 
en la producción escrita proponen la metacognición y la revisión como elementos fundamentales en el proceso de evaluación de la producción textual. En este grupo se destacan los trabajos de Gil (2010), Ribas (2006).

Los estudios, cuyas orientaciones teóricas se basan en la pedagogía crítica: Niño (2011), Díaz (2009), Arias (2009), destacan la necesidad de superar el rol instrumental de la evaluación, como instrumento de control, supervisión y medición. De igual manera, contribuyen a la comprensión de la evaluación en un intento por transformar las prácticas evaluativas en relación con los sujetos que hacen parte del proceso evaluativo y con la realidad histórica, social y cultural. Córdoba (2002) y Lodoño (2009), por su parte, intentan resignificar el papel del maestro y de los estudiantes en el proceso de transformación cultural, por lo cual cuestionan de manera contundente el papel de las pruebas estandarizadas y de los modelos conductistas y tecnológicos; los autores resaltan la importancia de la evaluación como práctica discursiva y pedagógica, y la definen como fundamental en los procesos de enseñanza-aprendizaje. Se interesan en los contextos políticos y sociales del lenguaje y el discurso en la sociedad. Las muestras se toman desde la enunciación de los interlocutores en el acto comunicativo.

\section{La evaluación de los aprendizajes en lengua materna desde una perspectiva crítica}

Se toman tres aspectos fundamentales para abordar la evaluación desde una perspectiva crítica: el conocimiento para la emancipación, la reflexión para la liberación y la importancia del discurso para la generación de identidad y poder.

En primer lugar, para la pedagogía crítica el conocimiento es el eje central para acceder a la libertad, de ahí su interés emancipador en la producción de conocimiento de los sujetos, intenta generar condiciones para que los grupos tomen conciencia de sus condiciones históricas y se conviertan en sujetos que producen conocimiento para la transformación social.

Desde el enfoque crítico Habermas (citado por Sverdlick, 2008) define tres intereses cognitivos básicos, técnico empírico-analítico y emancipador. El interés técnico que se orienta hacia el control y la gestión del medio y está en directa relación con el saber empírico-analítico, caracterizado por adoptar la forma de explicaciones causales de acontecimientos observables.

Por tanto, desde esta perspectiva, la evaluación de los aprendizajes prioriza en el control de los sujetos y se aleja de incluir procesos de autoevaluación y coevaluación para favorecer la permanente construcción de conocimiento por parte de los estudiantes evaluados. Restringe las posibilidades simbólicas del lenguaje a la instrumentalización de los aspectos formales de la lengua y a la constatación de saberes en términos de lo correcto y lo incorrecto. El interés emancipador se identifica con la autonomía de los sujetos y la responsabilidad; así que por medio de la evaluación es posible generar en los estudiantes la autorreflexión, si se les da la oportunidad de volver sobre sus dificultades y fortalecer sus aciertos en aras de permitir el acceso a un conocimiento que interprete la realidad social como una creación histórica relativa, que se puede construir y se puede transformar siempre que existan criterios evaluativos ligados a intencionalidades reales de los sujetos.

En segundo lugar, la pedagogía crítica nace en América Latina, se nutre del pensamiento pedagógico de Paulo Freire, quien consideraba a la educación como acto político, como acto de conocimiento y como acto creador. Para Freire, la liberación es el fin de la educación. Gadotti (2000) propone que "La educación tiene como fin la liberación, la transformación radical de la realidad para mejorarla, para volverla más humana, para permitir que hombres y mujeres sean reconocidos como sujetos de su historia y no como objetos" (p. 134). En este sentido, la evaluación debe propender a la generación de cono- 
cimiento en los sujetos para acortar las distancias sociales y permitir la construcción de sociedades más justas. De ahí la importancia de promover prácticas de autoevaluación y coevaluación como inicio de participación de los sujetos en su proceso de formación y autorregulación.

En tercer lugar, y frente a la importancia del discurso para la generación de identidad y poder, la pedagogía crítica concibe la escuela como el punto de convergencia entre la cultura del aula y la de la calle, donde confluyen diversas ideologías, formas sociales heterogéneas que luchan por la dominación. En consecuencia, surge la necesidad de pensar el lenguaje como praxis, de ver la importancia de la palabra y el discurso en la generación de identidades y de poder (Freire, 1979). En el marco de la palabra, McLaren (1998) afirma que "el lenguaje proporciona las autodefiniciones con las que las personas actúan, negocian diversas posiciones subjetivas y emprenden un proceso de nombrar y renombrar las relaciones entre ellas mismas, los otros y el mundo" (p. 49). Este planteamiento sugiere tensiones frente al uso de la palabra en el aula, la negociación de significados y las posibilidades de enunciación.

\section{La evaluación formativa}

Pensar los discursos y las prácticas evaluativas desde una perspectiva crítica implica ubicarse en el marco de la evaluación formativa, concebida como elemento que contribuye a la educación integral del estudiantado por cuanto promueve la potenciación significativa de la lengua y el lenguaje; reconoce los nexos entre la comunicación y la vida; contribuye a la formación de aspectos sociales, culturales, éticos, estéticos y axiológicos (Álvarez, 2001).

Es pertinente para este estudio comprender la manera cómo se evalúan los aprendizajes en el aula, los ambientes de interacción que se generan, los procesos argumentativos que se forjan, la forma como los participantes construyen sus conocimientos, el dialogo y la retroalimentación entre los sujetos de la evaluación. Lo anterior conlleva entender la eva- luación como una práctica pedagógica, indica que se trata de un proceso de construcción de conocimiento, de un proceso en el que se enseña y se aprende.

Un camino viable para asumir la evaluación desde una perspectiva crítica es la propuesta de evaluación formativa puesto que esta no pretende medir la cantidad de conocimiento adquirido, sino que establece relaciones entre las personas como sujetos de aprendizaje. Al respecto, Freire (1994) afirma que mejorar la calidad de la educación:

[...] implica la formación permanente de los educadores. Y la formación permanente consiste en la práctica de analizar la práctica. Pensando su prácti$\mathrm{ca}$, naturalmente con la presencia de personal altamente cualificado, es posible percibir imbuida en la práctica una teoría todavía no percibida, poco percibida o percibida pero poco asumida. (p. 91)

Una evaluación pensada al servicio del conocimiento y del aprendizaje de manera formativa constituye en sí misma un medio para el aprendizaje; la evaluación formativa va más allá de las pretensiones de calificar: "Evaluar con intención formativa no es igual a medir ni a calificar, ni tan siquiera a corregir... tampoco es clasificar ni examinar ni aplicar test, pero se confunde con ellos" (Álvarez, 2001, p.11). Así que la evaluación formativa no se centra en la corrección de errores, sino que pretende potenciar aciertos y actuar cuando aún es tiempo para mejorar.

Los principios base de la evaluación formativa abordan niveles cognitivos complejos que tienen en cuenta la retroalimentación para mejorar la construcción de conocimientos; para ello es indispensable no solo identificar cómo se produjo el resultado final del proceso, lo que llamamos evaluación sumativa, sino la evaluación se debe hacer de manera permanente, para valorar el avance cotidiano y así favorecer la retroalimentación.

\section{Sobre los discursos instruccionales: las consignas}

Para lograr una aproximación a la interpretación de los discursos se tienen en cuenta las consignas 
de los profesores. Desde la perspectiva de la evaluación formativa, las consignas son textos enmarcados en situaciones de comunicación; dichas situaciones presentan características comunes en las que el profesor, mediante su discurso instruccional, organiza unos contenidos e intenta que los estudiantes realicen ciertas actividades. Respecto a la consigna Riestra (2010) afirma que "es el texto que organiza las acciones mentales en los aprendientes; podemos decir, el que regula, el que ordena, el que dirige, el que prescribe, etc." (p. 178). Por estas consideraciones, las consignas constituyen una unidad de análisis de la propuesta.

Tomando en cuenta lo precedente, mediante sus consignas, el profesor posibilita ya sea la construcción de conocimientos o la repetición de contenidos. A la luz de los planteamientos de Riestra (2010), para que esta funcione como instrumento de la organización semántica de la conciencia debe operar como un andamiaje del pensamiento. Esto significa que el profesor con sus consignas debe operar en la zona de desarrollo próximo del estudiante para facilitar la construcción de conocimientos. Esto es posible si se plantean actividades que sugieren la progresión de procesos cognitivos y el aumento de niveles de dificultad en la elaboración de la tarea; si, por el contrario, el profesor propone actividades que no requieren procesos mentales complejos, limita las posibilidades de los estudiantes para construir competencias y habilidades para resolver problemas y necesidades $\mathrm{y}$, a su vez, limita la construcción de aprendizajes autónomos. De esta manera, la consigna supone un plano práctico en cuanto la planificación de una tarea y del mismo modo un plano teórico, por cuanto refleja las concepciones de los docentes, en este caso, sobre los conocimientos del lenguaje.

Tomando las directrices de la evaluación formativa no es suficiente una calificación respecto a si las respuestas son correctas o incorrectas, sino que, para facilitar el aprendizaje, es necesario que haya criterios claros de desempeño y que se les propor- cione a los estudiantes estrategias de mejoramiento. Esto no es posible si las prácticas evaluativas se reducen a la calificación cuantitativa o a la acumulación de revisados que en sí mismos no le comunican al estudiante su nivel de desempeño de habilidades o competencias específicas. En este caso el conocimiento estaría apartado de los sujetos, funcionaría como una entidad aislada de la vida humana y como tal no se pueden lograr transformaciones en la conciencia ni en las relaciones interpersonales situadas en un contexto histórico y cultural.

\section{Sobre los principios de clasificación y enmarcación}

El discurso pedagógico hace parte del dispositivo pedagógico, el cual por medio de reglas de distribución, de recontextualización y de evaluación, distribuye el poder, el conocimiento y las formas de conciencia. Las reglas de distribución son las que "regulan la especialización diferente de la conciencia de diversos grupos" (Bernstein, 1990, p. 126) y están en directa relación con el principio de clasificación. Esto significa que el dispositivo pedagógico decide quién puede transmitir, qué se puede transmitir, a quién y en qué condiciones se puede transmitir.

Bernstein (1990) agrega que la consolidación de códigos está mediada en gran medida por la educación, ella se encarga de reproducir las estructuras sociales existentes mediante la aplicación de los principios de clasificación y enmarcación. Los códigos sociolingüísticos como educativos "se generan, reproducen y cambian como resultado de los rasgos macro institucionales de la sociedad y cómo se generan, reproducen y cambian en los niveles más específicos de interacción tanto en la familia como en la escuela" (p. 3). Los códigos, elaborados y restringidos implican reglas de reconocimiento y presuponen los principios de clasificación y enmarcación; esta afirmación conduce a pensar la existencia de relaciones de poder y principios de control, por lo cual se considera que el análisis de la enmarcación y la 
clasificación posibilita hacer visibles las maniobras de poder y control que se dan en las instituciones educativas y al mismo tiempo hay una "relación de interdependencia entre macro estructuras y micro prácticas de interacción y comunicación" (Bernstein, 1990, p. 4).

El principio de clasificación, que se relaciona con la reglas de distribución del dispositivo pedagógico y con el discurso instruccional del discurso pedagógico es el que regula la relación entre la distribución del poder, la distribución del conocimiento y de conciencia. Estos aspectos se tendrán en cuenta en el análisis de los discursos y las prácticas de los docentes de lengua castellana. Con ello se intenta observar e interpretar la manera como es distribuido el poder, el conocimiento y la conciencia en un escenario particular de educación formal en situaciones de evaluación.

Es importante tener en cuenta el principio de la enmarcación entendido como:

[...] principio que regula las relaciones sociales, esto es, principio que subyace a las prácticas de comunicación. La enmarcación nos remite a la comunicación y a las relaciones de poder manifiestas en la interacción. La enmarcación constituye, legítima y mantiene la estructura de las relaciones comunicativas en las que está presente siempre el control. (Bernstein, 1990, p. 21)

Esto significa que hay un vínculo entre la enmarcación y el discurso regulativo. Si el interés es interpretar las prácticas pedagógicas relacionadas con la evaluación, no es posible el distanciamiento con el principio de la enmarcación, sugerido por Bernstein (1990), ya que es este el que "regula las prácticas pedagógicas de las relaciones sociales creadas por el proceso de reproducción del conocimiento educativo" (p.23).

El profesor hace uso de una enmarcación fuerte cuando explícitamente regula los principios de interacción, es decir, da órdenes, organiza espacialmente, ordena posturas específicas, entre otros. Del mismo modo, el maestro utiliza una enmarcación débil en la que no es explícita la regulación de la interacción, por lo tanto el estudiante tiene un mayor control sobre los principios comunicativos. En este caso, el estudiante tiene un nivel de autonomía mayor que en la enmarcación fuerte.

La unión de la clasificación y la enmarcación dan cuenta del código y constituyen su gramática. "Las relaciones de poder, constituyen, legitiman y mantienen las clasificaciones del código (rasgos paradigmáticos) y las relaciones de control, constituyen mantienen y legitiman la enmarcación de las relaciones comunicativas (Rasgos sintagmáticos)" (Bernstein, 1990, p. 21). Los rasgos sintagmáticos, como formas de distribución y clasificación de conocimiento, y los rasgos paradigmáticos, entendido como control y enmarcación, se constituyen en objeto de análisis para la interpretación de las prácticas evaluativas instauradas al interior del aula de lengua materna.

\section{Metodología}

La investigación se desarrolla en el marco del paradigma interpretativo que permite estudiar los fenómenos desde lo descriptivo y desde la perspectiva de los sujetos para interpretar la realidad observada y conocer la manera cómo los actores del sistema significan el mundo social que constituyen (Hernández, 2006). El enfoque es cualitativo, permite significar la realidad a partir del conocimiento, las actitudes y los comportamientos (Guba y Lincon, 1994). El diseño corresponde al estudio de caso propuesto por Stake (1999) como un análisis integral, una indagación empírica que investiga un fenómeno contemporáneo dentro de su contexto en la vida real. Los sujetos del caso en estudio son cuatro profesores, licenciados en lengua castellana que laboran en una institución educativa oficial de Cundinamarca, en educación básica.

Los datos se obtienen mediante observaciones directas, entrevistas semiestructuradas, conversaciones 
informales, cuadernos de los estudiantes, grabaciones de clase en audio y video. Se establecen como unidades de análisis los discursos y las prácticas evaluativas, para los discursos las categorías de análisis corresponden a las consignas, las categorías teóricas de análisis corresponden a los principios de clasificación y enmarcación.

La herramienta utilizada es el análisis de contenido semántico, Bardin (1996) lo conceptualiza como:

[...] el conjunto de técnicas de análisis de las comunicaciones tendientes a obtener indicadores (cuantitativos o no) por procedimientos sistemáticos y objetivos de descripción del contenido de los mensajes, permitiendo la inferencia de conocimientos relativos a las condiciones de producción/recepción (contexto social) de estos mensajes". (p. 32)

Se vale de matrices semánticas para establecer relaciones entre los significados, codificar, categorizar, realizar inferencias y generalizaciones tras un proceso de lectura sistemática e interpretativa de los datos.

\section{Análisis de datos}

Los discursos evaluativos forman parte del discurso pedagógico, son directivas de trabajo que el profesor imparte cuando desarrolla su tarea. Estas directivas son denominadas por Dora Riestra como consignas de trabajo (2010). En este estudio se toman las consignas para describir, caracterizar y analizar los discursos evaluativos que circulan en el aula. Se inicia el análisis con un listado de 300 consignas obtenidas de los cuadernos de los estudiantes que luego se redujeron a 145 por su carácter prototípico.

Para leer los datos se elabora una matriz semántica orientada a establecer la función evaluativa que cumplen las consignas, la frecuencia con que se manifiestan, para de esta manera establecer algunas categorías emergentes. En la tabla 1 se visualiza el proceso hasta aquí descrito.

La frecuencia y la interacción de los datos establecen que el uso mayoritario corresponde a los discursos cuya función se ubica en el control de las acciones; dentro de este grupo se encuentran las categorías que inicialmente se denominan: control, cumplimiento, forma, memoria y copia.

Tabla 1. Categorías en relación con la función evaluativa de las consignas y frecuencia

\begin{tabular}{|l|c|}
\hline Categoría & Frecuencia \\
\hline Control & 86 \\
\hline Cumplimiento & 28 \\
\hline Copia & 12 \\
\hline $\begin{array}{l}\text { Creación a partir } \\
\text { de lo conocido }\end{array}$ & 12 \\
\hline Forma & 05 \\
\hline Interacción & 05 \\
\hline Memoria & 04 \\
\hline Opinión & 03 \\
\hline Total & 145 \\
\hline
\end{tabular}

Fuente: elaboración propia.

Si la consigna, según las ideas de Riestra (2010) "es el texto que organiza las acciones mentales de los estudiantes y al mismo tiempo, es la que organiza la acción que cada alumno internaliza” (p. 179), significa que los estudiantes, en este caso particular, internalizan acciones que se enmarcan en el cumplimiento de las tareas, bajo situaciones de control y proceden de acuerdo con las directrices dadas por los profesores, siguen órdenes establecidas y cumplen con las tareas propuestas.

Los resultados anteriores llevan a establecer nuevas relaciones semánticas para a agrupar las consignas por la función que cumplen. La síntesis se presenta de manera explícita en la tabla 2.

El análisis de la función que cumplen las consignas permitió establecer relaciones metafóricas del ámbito del aula de clase con un escenario teatral, este se describe más ampliamente en los siguientes apartados. 
Tabla 2. Matriz semántica para agrupar consignas por la función que cumplen

\begin{tabular}{|l|l|}
\hline \multicolumn{1}{|c|}{ La función que cumplen las consignas } & \multicolumn{1}{c|}{ Grupo de consignas } \\
\hline Control & Control, cumplimiento, forma, memoria y copia \\
\hline Interacción & Creación, opinión e interacción \\
\hline
\end{tabular}

Fuente: elaboración propia.

\section{Control y cumplimiento: protagonistas en el escenario del aula de lengua materna}

Los datos comunican que las actividades propuestas no requieren una evidente reorganización semántica por parte de los estudiantes; se constituyen más en formas de reproducción de contenidos y definiciones. Copiar y memorizar se convierten en el guion didáctico que representan los actores, guiados por el director de la escena pedagógica. Los estudiantes permanentemente realizan actividades psicomotrices como recortar, dibujar, pegar, subrayar, identificar, transcribir y completar para cumplir con las indicaciones dadas por el profesor, quien, a manera de director, verifica que los personajes sigan literalmente el guion que ha sido escrito para ellos. Esta situación generó la reflexión acerca de la relación entre las actividades predominantes (copiar, subrayar, colorear, identificar, redactar, cumplir con el orden, etc.) y los procesos de organización semántica necesarios en la construcción de conocimiento. Según los planteamientos de Vygotsky y referenciados por Riestra (2010), para que la consignas se conviertan en instrumentos de la organización semántica de la conciencia de los estudiantes, deben operar como andamiaje del pensamiento, para actuar en la zona de desarrollo próximo.

\section{La interacción: un intento para la creación}

Los datos muestran también que en una proporción reducida están los discursos que se orientan a la creación, a la opinión y a la interacción; esto lleva a pensar que las situaciones de comunicación, de manera limitada, hacen parte del escenario del aula. En ocasiones los profesores se distancian de los guiones prefabricados en el contexto escolar para dar la palabra a los actores principales de la escena, los estudiantes. Los sueños, los temores, las vivencias y los deseos se convierten en palabras que cobran vida cuando son escuchadas por otros en escenarios de interlocución. Los escenarios son creados por los profesores en el momento en que permiten que los estudiantes se comuniquen desde lo que piensan, sienten y desean, motivados por intencionalidades $y$ propósitos auténticos y cuando el error es utilizado de manera consciente para la construcción de personajes propios que hacen parte de un solo escenario.

\section{Las consignas en relación con la enseñanza de la lengua}

Una vez discutido el para qué se utilizan las consignas, conviene preguntar qué conocimientos propios del lenguaje se desarrollan por medio de los discursos de los profesores. Las categorías definidas anteriormente permitieron establecer, de manera general, que la mayoría de los discursos están orientados a la enseñanza de la lengua y en menor proporción a la enseñanza de la literatura; más adelante se explicará la función que cumplen las consignas en la enseñanza de la literatura. En la tabla 3 se observan las categorías resultantes de la función que cumplen las consignas en relación con la enseñanza de la lengua.

\section{El carácter prescriptivo de la lengua}

Los anteriores datos permiten identificar una amplia gama de instrucciones orientadas a la escritura y la lectura desde una perspectiva normativa, que atiende exclusivamente a los aspectos formales de la estructura superficial de la lengua. Los conocimientos que se imparten contribuyen muy poco al desarrollo de competencias en la comprensión y producción textual. 
Tabla 3. Matriz semántica para establecer categorías en relación con la función de las consignas en la enseñanza de la lengua

\begin{tabular}{|l|l|}
\hline $\begin{array}{c}\text { Categorías orientadas a la función que } \\
\text { cumplen las consignas en la ensenanza de la lengua }\end{array}$ & \multicolumn{1}{c|}{ Grupo de consignas } \\
\hline Carácter prescriptivo & $\begin{array}{l}\text { Identificación de categorías gramaticales, prelación } \\
\text { de aspectos formales, transcripción de definiciones y } \\
\text { lectura a nivel literal. }\end{array}$ \\
\hline Carácter pragmático & $\begin{array}{l}\text { Producción de textos a partir de títulos y situaciones } \\
\text { auténticas. }\end{array}$ \\
\hline
\end{tabular}

Fuente: elaboración propia.

La enseñanza de la lectura y la escritura desde una perspectiva prescriptiva procura la adquisición de destrezas cuyos resultados se prevén y miden, de acuerdo con un patrón preestablecido, sin generar procesos de reflexión. De ahí que es pertinente clasificar las actividades de escritura hasta aquí descritas como una forma de racionalidad técnica, de acuerdo con lo expresado por Giroux (1998, p.124).

\section{El carácter pragmático de la lengua}

De igual forma, los datos permiten interpretar que, aunque en menor proporción, en el aula también circulan discursos instruccionales distantes del carácter instrumental de la modalidad escrita. Escribir lleva consigo la configuración de sentido, implica comprender que este acto sirve para algo, es útil y produce efectos de recepción en los lectores. Aquí la función de la escritura es entendida como una construcción narrativa de la realidad y está en concordancia con la modalidad narrativa del pensamiento enunciada por Bruner (2004). Por cuanto es un proceso de subjetivación de lo real que consiste en construir la experiencia del mundo mediado por la subjetividad.

Los discursos instruccionales con orientación pragmática tienen características de lo que Eisner (1995) denominó la inteligencia cualitativa. Esta no se caracteriza como algo innato, sino como modo de acción humana que puede crecer por medio de la experiencia y es capaz de emplearse en el espectro más amplio de la vida de los sujetos, como percepción, comprensión y creación de múltiples realidades. Esto conduce a pensar que en el aula de lengua también circulan discursos que van más allá del uso repetitivo y mecánico de los códigos con que lo real se configura y comunica.

\section{Enseñanza de la literatura}

La lectura y la escritura, en relación con el discurso literario, se plantea en dos direcciones: en primer lugar, la escritura se inscribe en lo que se denomina espontaneidad creadora y, en segundo lugar, tanto la lectura como la escritura literaria se supeditan a la teoría lingüística. Es así como las actividades propuestas en las consignas propician un distanciamiento de las características y esencia del texto literario, esto indica que lo literario no ha alcanzado su independencia y autonomía, por cuanto se aborda desde las lógicas de la lingüística estructural y no desde sus propias lógicas retóricas.

La escritura del texto literario se sugiere a partir de la creación espontánea, por cuanto en las consignas no se hacen precisiones ni restricciones retóricas. Bajo estas premisas para escribir literatura solo se requiere disponibilidad de tiempo. De manera implícita, al estudiante se le limita la posibilidad de comprender las particularidades inherentes a cada tipo de discurso literario; asimismo se le impide que cualifique sus experiencias escriturales porque no se le brinda la oportunidad de volver sobre su tex- 
to, para dialogar con él y para conocer sus propias limitaciones y alcances como potenciales escritores de ficción literaria.

La ficción exige de lógicas que son parte del aprendizaje escolar, mediante la lectura y la escritura de ficción se tiene en cuenta el género elegido, la situación de comunicación, los posibles destinatarios y los objetivos; en otras palabras, se da cuenta de la dimensión retórica de la literatura. Desde esta perspectiva, la escritura sobrepasa la consideración de mero instrumento de comunicación y se asume como un acto que permite la producción de conocimiento ya que cuando se escribe, implícita o ex- plícitamente, se aprende algo que no se sabía. Es así como la escritura se constituye en un instrumento didáctico que el maestro debe potenciar mediante consignas que hagan restricciones retóricas para que los estudiantes comprendan la lógica de las ficciones literarias mediante el ejercicio mismo de la escritura.

\section{Discurso instruccional y clasificación}

Teniendo en cuenta lo planteado hasta el momento y de acuerdo con los principios de clasificación, el interés se centra ahora en comprender la relación entre poder, conocimientos y formas de conciencia. Estas relaciones se aprecian mejor en la tabla 4.

Tabla 4. Matriz semántica para establecer relaciones entre consignas, respuestas de los niños y prácticas evaluativas

\begin{tabular}{|c|c|c|}
\hline Consigna (discurso) & Respuesta del niño & Práctica evaluativa \\
\hline $\begin{array}{l}\text { Lee un texto y copia un } \\
\text { ejemplo de narración } \\
\text { en tercera persona. } \\
\text { Lee un texto y copia un } \\
\text { ejemplo de narración } \\
\text { en primera persona. } \\
\text { Invento y escribo una } \\
\text { historia en primera } \\
\text { persona }\end{array}$ & $\begin{array}{l}\text { Érase una vez una hermosa gallina que vivía muy holgada- } \\
\text { mente en su cortijo, rodeada de su numerosa familia, en la } \\
\text { cual se distinguía un pollo deforme y estropeado. } \\
\text { Por primera vez he visto un cadáver, es miércoles y por } \\
\text { primera vez siento como si fuera domingo porque no he ido } \\
\text { a la escuela y me he puesto este vestido que me aprieta en } \\
\text { alguna parte. } \\
\text { Érase un niño llamado Anderson. Cuando yo estaba en pro- } \\
\text { niño y la profe tenemos un paseo a Bogotá, es una sorpresa, } \\
\text { nos fuimos en el bus cantando y todos jugaban cuando salí } \\
\text { del bus vi un hermoso parque y grande hay nos dieron un } \\
\text { sándwich con un vaso de gaseosa entramos, todos se reu- } \\
\text { nieron en un bosque hermoso, la profe nos dijo a las } 12: 30 \\
\text { nos vemos para darle el almuerzo... }\end{array}$ & $\begin{array}{l}\text { El profesor coloca } \\
\text { revisado (chulo) y un } \\
\text { número que correspon- } \\
\text { de al consecutivo de la } \\
\text { actividad presentada. } \\
\text { No hace ninguna obser- } \\
\text { vación. }\end{array}$ \\
\hline
\end{tabular}

Fuente: basada en el registro tomado de los cuadernos de grado octavo.

En la siguiente matriz semántica (tabla 5) se observan los conocimientos que se distribuyen, la fuerza de la clasificación y los efectos performativos.

De acuerdo con el principio de clasificación, el profesor, mediante su consigna, está distribuyendo unos conocimientos: existen narradores en primera y en tercera persona, pero el hecho de no propiciar procesos de configuración de sentido impide que el estudiante comprenda la narrativa como forma de subjetividad y como discurso que hace parte de su propia existencia. El profesor distribuye un conocimiento sin que este genere afectaciones de tipo afectivo ni cognitivo, limita al estudiante la posibilidad y el poder de usar la lectura y la escritura como formas de empoderamiento en situaciones de comunicación. Más bien se está promoviendo en él el copiado de textos, ya que la lectura y la escritura se abordan como acciones que no revierten altos niveles de complejidad y que se distancian de intencionalidades auténticas tanto de los lectores como de los productores de textos. 
Tabla 5. Matriz semántica para establecer relaciones entre los conocimientos distribuidos, la fuerza de la clasificación y los efectos performativos

\begin{tabular}{|l|c|l|}
\hline Conocimientos distribuidos & Fuerza de la clasificación & \multicolumn{1}{|c|}{ Efectos performativos } \\
\hline Leer para identificar & C- & Realización de actos mecánicos \\
\hline Copiar un texto & C- & $\begin{array}{l}\text { Realización de actos mecánicos } \\
\text { de transcripción }\end{array}$ \\
\hline
\end{tabular}

Fuente: elaboración propia.

Las consideraciones anteriores llevan consigo unas formas propias de situar a los estudiantes en contextos de comunicación en las que el lenguaje es un objeto abstracto, conformado por reglas y que el conocimiento de estas garantiza su correcta ejecución. En este caso, la narración es una forma de comunicación sin interlocutores reales, que se enseña mediante de la identificación de los tipos de narrador. Esto significa que la clasificación de los profesores es débil, en términos de grado de profundidad y especialización de habilidades y competencias propias del lenguaje.

\section{Discurso regulativo y enmarcación}

A partir de los discursos que circulan en el aula se observa que el control y el cumplimiento se constituyen en los protagonistas del escenario pedagógico. Esto lleva a considerar ciertas formas de comunicación en el aula en relación con el discurso regulativo y la enmarcación. Inicialmente se toman ejemplos para establecer la manera cómo opera el discurso regulativo en el plano pedagógico, para este efecto se centra la atención en la práctica evaluativa del docente, en relación con la consigna y los conocimientos. El procedimiento se evidencia en la tabla 6.

Tabla 6. Matriz semántica para analizar el discurso regulativo

\begin{tabular}{|c|c|c|}
\hline Consigna (discurso) & Respuesta del niño & Práctica evaluativa \\
\hline $\begin{array}{l}\text { Lee un texto y copia un ejem- } \\
\text { plo de narración en tercera } \\
\text { persona. } \\
\text { Invento y escribo una historia } \\
\text { en primera persona }\end{array}$ & $\begin{array}{l}\text { El niño copia el ejemplo de narración en tercera } \\
\text { persona. } \\
\text { Érase un niño llamado Anderson. Cuando yo } \\
\text { estaba en proniño y la profe tenemos un paseo } \\
\text { a Bogotá, es una sorpresa, nos fuimos en el bus } \\
\text { cantando y todos jugaban cuando salí del bus } \\
\text { vi un hermoso parque y grande hay nos dieron } \\
\text { un sándwich con un vaso de gaseosa entramos, } \\
\text { todos se reunieron en un bosque hermoso, la } \\
\text { profe nos dijo a las 12:30 nos vemos para darle } \\
\text { el almuerzo... }\end{array}$ & $\begin{array}{l}\text { El profesor coloca revisado } \\
\text { (chulo). No hace ninguna } \\
\text { observación. El profesor en } \\
\text { su práctica lleva un conse- } \\
\text { cutivo numérico de las ac- } \\
\text { tividades presentadas por } \\
\text { el estudiante. Al finalizar el } \\
\text { periodo académico por cada } \\
\text { revisado que le falte se res- } \\
\text { tan tres décimas a su nota. }\end{array}$ \\
\hline
\end{tabular}

Fuente: elaboración propia basada en el registro tomado de cuaderno de grado octavo.

La práctica evaluativa del profesor garantiza la presentación de la actividad, pero no genera en el estudiante procesos de metacognición mediante la revisión. Existe una enmarcación fuerte en lo formal de la tarea, como el color de la tinta que se debe usar, las características de las hojas y el número de cuartillas, pero no se hace ninguna indicación respecto a la producción textual de la narración. Esto significa que hay mayor interés por el cumplimiento de la actividad que por el proceso de producción literaria. 
De esta manera, el profesor prioriza en lo regulativo de la tarea antes que lo instruccional de esta.

En la mayoría de las consignas analizadas se observa que el control se ejerce mediante las prácticas evaluativas recurrentes: la calificación numérica y la acumulación de sellos, chulos y firmas. En los registros no aparecen observaciones de los profesores con respecto a las respuestas de los niños, es decir, no existe un proceso de retroalimentación. Lo descrito se ubica en formas de comunicación unidireccionales, la voz del estudiante se desconoce y la del maestro se traduce en ordenar, por tanto, se puede inferir que la función específica de la práctica evaluativa de los profesores observados es de verificación del cumplimiento de una tarea. Mediante la fuerza de la enmarcación se puede establecer cómo sucede la comunicación en el aula. Hay enmarcación fuerte de la interacción cuando el profesor ejerce explícitamente formas de comunicación unidireccionales y verticales. La fuerza de la enmarcación es débil cuando se propende a situaciones de comunicación horizontales. En la tabla 7 aparece una matriz semántica para explicar mejor cómo opera la fuerza de la clasificación.

Tabla 7. Matriz semántica para establecer relaciones entre comunicación en el aula, fuerza de la enmarcación y efectos performativos

\begin{tabular}{|l|c|l|}
\hline $\begin{array}{c}\text { Prácticas evaluativas. Comunicación } \\
\text { en el aula }\end{array}$ & $\begin{array}{c}\text { Fuerza de la } \\
\text { Enmarcación }\end{array}$ & \multicolumn{1}{c|}{ Efectos performativos } \\
\hline $\begin{array}{l}\text { Énfasis en la forma de la tarea: color } \\
\text { de tinta, número y tamaño de las } \\
\text { hojas. }\end{array}$ & E+ & $\begin{array}{l}\text { Interiorización de aspectos formales mas no } \\
\text { de conocimientos. }\end{array}$ \\
\hline Acumulación de firmas, sellos, chulos. & E+ & $\begin{array}{l}\text { Cumplimiento sin reflexión sobre aciertos o } \\
\text { dificultades. } \\
\text { Comunicación unidireccional }\end{array}$ \\
\hline
\end{tabular}

Fuente: elaboración propia.

La matriz anterior permite establecer que las características de la comunicación en el aula se circunscriben en la relación emisor-receptor. El emisor (docente) es quien sabe, controla y ordena al receptor (estudiante), quien oye y cumple las órdenes. Es unidireccional en la medida en que la información va del profesor hacia el estudiante, lo cual lleva en su interior matices de verticalidad y autoritarismo; el profesor por medio de sus discursos y prácticas evaluativas se sitúa como poseedor de la verdad y le atribuye al estudiante el papel de receptor de conocimientos.

La relación comunicativa anteriormente descrita se asocia de manera directa con lo que Bernstein denomina discurso regulativo, el cual hace que se institucionalice una relación distante entre el profesor y el estudiante. En este sentido, según los planteamientos de Bernstein, el problema radica en la distancia que hay entre el contexto de producción de conocimiento científico y el contexto de reproducción o aprendizaje académico. En este orden de ideas, la escuela no es un contexto significativo para el aprendizaje, toda vez que desconoce los problemas relevantes de la comunidad y se configura como una estructura artificial, alejada de la vida de los estudiantes.

A continuación se presenta la discusión de resultados del caso en estudio y algunas reflexiones a manera de conclusión. 


\section{Discusión de resultados y conclusiones}

Por medio del proceso investigativo se observó que el discurso pedagógico de tres de los docentes prioriza en lo regulativo, cuya función principal se centra en el control de las actividades propuestas, en términos de cumplimiento. Esto refleja que en el aula de lengua materna está presente la enmarcación fuerte, materializada en las prácticas evaluativas que sugieren el cumplimiento más que el desarrollo de habilidades y competencias propias de las ciencias del lenguaje. Así, la interacción entre docentes y estudiantes se supedita a la verificación y al control.

La evaluación manifiesta en los discursos y las prácticas refleja que las relaciones pedagógicas se ven influenciadas por el poder regulativo que se le ha otorgado a los docentes en su formación académica. Ellos hacen parte de un sistema social en el que su labor se ubica en la formación de sujetos que deben cumplir con unos requerimientos específicos de las demandas económicas y sociales. En este caso, el discurso instruccional, en términos de desarrollo de competencias del lenguaje, se supedita al discurso regulativo.

Los conocimientos que se distribuyen no alcanzan la profundidad necesaria para consolidar en los estudiantes niveles de conciencia que permitan la reflexión acerca de su entorno, y de su posible transformación con miras a alcanzar relaciones sociales más justas y equitativas. La enseñanza del lenguaje se ubica en los niveles estructurales, es muy reducido el desarrollo de competencias centradas en las dimensiones afectiva, comunicativa y cognitiva.

La mayoría de los discursos de los profesores cumplen con la función de dirigir las acciones y los comportamientos de los estudiantes mediante actividades que distan del desarrollo de procesos cognitivos superiores que le otorguen al alumno la posibilidad de cuestionamientos sobre su propia realidad y la de su entorno. Más bien se enfatiza en la repetición de contenidos y definiciones; acciones que se relacionan con una tendencia prescriptiva de la lengua.
Es precario el trabajo del lenguaje desde una perspectiva pragmática, por lo tanto, la interacción es claramente limitada en el aula de lengua materna. Paradójicamente, en la enseñanza del lenguaje se desarrolla muy poco la comunicación humana. Esta situación obedece a la ubicación social del maestro, quien es el encargado de establecer el orden y el control de las actuaciones de los estudiantes. De allí que el discurso oral es trabajado en menor proporción que el escrito, ya que el primero puede interferir de manera directa con el orden establecido en el aula.

De acuerdo con lo anterior, se observa que el orden y el control son los protagonistas de la escena pedagógica en la que lo más importante no es la enseñanza de la lengua, sino el cumplimiento y la verificación de la tarea. Los discursos de los profesores, en este caso, se sitúan en la tradición estructuralista del lenguaje en la que prima lo prescriptivo sobre lo comunicativo.

No obstante, se observa que en al aula también circulan discursos instruccionales distantes del carácter instrumental de la escritura. Se evidencia que escribir lleva consigo la configuración de sentido, implica comprender que este acto sirve para algo, es útil y produce efectos de recepción en los lectores. Este tipo de relación pedagógica distribuye conocimientos que le otorgan al estudiante el poder de usar el lenguaje para pensar, discutir, proponer, crear, comunicar, es decir, para ser y estar en el mundo.

La clasificación de los profesores es débil, por cuanto solo se abordan algunas de las características del lenguaje y se le desconoce como elemento transformador de la experiencia humana, paralelamente se limita al estudiante la posibilidad de comprender el mundo y comunicar ideas nuevas a los demás.

El profesor prioriza en lo regulativo de la tarea sobre lo instruccional de esta. El discurso instruccional está incluido en el regulativo; así, el discurso pedagógico no se produce a partir de las competencias y las habilidades que deben desarrollarse en el área 
de lengua castellana, sino a partir de la regulación de las acciones y las conductas, y dentro del discurso instruccional se ordena la habilidad que se debe adquirir.

\section{Reconocimientos}

El artículo presenta resultados de la investigación «Discursos y prácticas evaluativas en aula de lengua materna: un estudio de caso». Realizada para obtener el título de Magister en Pedagogía de la Lengua Materna de la Universidad distrital Francisco José de Caldas.

\section{Referencias}

Álvarez, J. (2001). Evaluar para conocer, examinar para excluir. Madrid: Morata.

Arias, N. (2009). Para el debate en torno a la investigación en evaluación de aprendizajes. Itinerario Educativo, 54, 73-96.

Atorresi, A. (2006). Segundo Estudio Regional Comparativo y Explicativo (SERCE). Manual para la evaluación de las pruebas de escritura. Argentina: Oficina Regional de Educación, Unesco para América Latina y el Caribe y el Laboratorio Latinoamericano de Evaluación de la Calidad de la Educación (LLECE).

Aterrosi, A. (2009). Segundo Estudio Regional Comparativo y Explicativo (SERCE). Aportes para la enseñanza de la lectura. Chile: Oficina Regional de Educación Unesco para América Latina y el Caribe y el Laboratorio Latinoamericano de Evaluación de la Calidad de la Educación LLECE.

Bardin, L. (1996). Análisis de contenido (2a ed.). Madrid: Akal.

Bernstein, B. (1990). La construcción social del discurso (Mario Díaz, Trad.). Bogotá: Prodic.

Bruner, J. (2004). Realidad mental y mundos posibles. Los actos de la imaginación que dan sentido a la experiencia. Barcelona: Gedisa.
Camargo, M., Gutiérrez, M. y Pedraza, A. (2006). Obstáculos para el uso institucional de la evaluación. Revista Educación y Educadores, 9(2), 23-32.

Camelo, J. (2010). Las consignas como enunciados orientadores de los procesos de escritura en el aula. Enunciación, 15(2), 58-69.

Camargo, Z., Uribe, G. y Caro, M. (2010). Evaluación de una muestra representativa de los relatos presentados al Concurso Nacional de Cuento Gabriel García Márquez (2007). Enunciación, 15(1), 78-94.

Castrillón, C., Camargo, Z., y Caro, M. (2010). La evaluación de la escritura en el marco de un concurso: del texto narrativo al cuento literario. Enunciación, 15(1), 7-16

Córdoba, G. (2002). Exclusión y resistencia cultural. Pensamiento actual, 10(4), 10-16. Recuperado el 16 de septiembre de 2012, de http://www.revistas.ucr.ac.cr/index. php/ pen samiento-actual/ $\operatorname{article/view/8235,~}$

Díaz, A. (2009). La evaluación universitaria en el contexto del pensamiento neoliberal. Mientras tanto, 110/111, 67-86. Recuperado el 13 de marzo de 2013, de http://www.jstor.org/stable/27821334

Eisner, E. (1995). Educar la visión artística. Barcelona: Paidos.

Freire, P. (1979). Educación y cambio. Argentina: Ediciones Búsqueda.

Freire, P. (1994). Educación y participación comunitaria. En M. Castells, R. Flecha, P. Freire, H. Giroux, D. Macedo y P. Willis (Eds.), Nuevas perspectivas críticas en educación (pp. 83-97). Barcelona: Paidós.

Gadotti, M. (2000). Perspectivas actuales de la educación. Buenos Aires: Siglo XXI.

Gil., J. (2010). Enseñar y evaluar textos narrativos. Algo más que puro cuento. Enunciación, 15(1), 56-77. 
Giroux, H. (1998). La escuela y la lucha por la ciudadanía. México: Siglo XXI editores.

Guba, E. y Lincoln, Y. (1994). Criterios de credibilidad en la investigación naturalista. En J. Gimeno Sacristán y A. Pérez Gómez (Eds.). La enseñanza: su teoría y su práctica (3a ed.) (pp. 148-165). Madrid: Akal.

Hernández R., Fernández, C. y Baptista, P. (2006). Metodología de la investigación. México: .Mc. Graw Hill.

Herrera, P., Gámez, P., Torres, A., Corredor, J. \& Quintero, F. (2008). ¿Qué pensamos cuando evaluamos? La evaluación al tablero. Enunciación, 15(1), 38-44.

Jurado, F. (2003). La evaluación externa en las áreas de lenguaje, matemáticas, ciencias y lengua extranjera. En Autor (Coord.). Evaluación: conceptualización, experiencias, prospecciones (pp. 4786). Bogotá: Universidad Nacional de Colombia.

Jurado, F. (2005) El concepto de competencia en los contextos de evaluación externa: un estudio de caso. Revista Enunciación, 10(1), 22-30.

Jurado, F. (Coord.). (2009). Los sistemas nacionales de evaluación. América Latina: ¿Impacto pedagógico u obediencia institucional? Bogotá: Universidad Nacional de Colombia.

Londoño, J. (2009). El poder del discurso y el discurso del poder. Entrevista a Teun A. Van Dijk. Revista de Lingüística y Lenguas Aplicadas, 4, 257-267.

Martínez, F. (2009). Evaluación formativa en aula y evaluación a gran escala: Hacia un sistema más equilibrado. Revista Electrónica de Investigación Educativa, 11(2). Recuperado de http://redie. uabc.mx/
Mclaren, P. (1998). Pedagogía, identidad ypoder. Argentina: Homo Sapiens.

Niño, L. (2011). Evaluación docente y currículo: desde la perspectiva evaluativa y curricular instrumental a la perspectiva crítica. Bogotá: Universidad Pedagógica Nacional.

Pérez, M. (2007). La evaluación externa de la producción escrita en Bogotá (2005-2007): más allá de la búsqueda del déficit en los estudiantes. Enunciación, 15(2), 17-31.

Riestra, D. (2010). Lectura y escritura en la universidad: las consignas de tareas en la planificación de la reenseñanza de la lengua. Enunciación, 15(1), 175-183.

Rodríguez, M. (2010). Exploraciones en torno a concepciones de docentes sobre la evaluación. Enunciación, 15(1), 36-58.

Saavedra, A. (2010). Evaluación de la creación literaria: de la racionalidad técnica tradicional al enfoque crítico, formativo y artístico. Enunciación,15(1), 118-133.

Stake, R. (1999). Investigación con estudio de casos. Madrid: Morata.

Sverdlick, Ingrid. (2008). De Freire a nosotros y de nosotros a Freire. Revista Novedades Educativas, (209 - 2008). Recuperado el 12 de marzo de 2013, de http://www.google.com.co/search?sour ceid $=$ navclient $\&$ ie $=U T F 8 \& r l z=1$ T4AURUenCO $502 \mathrm{CO} 503 \& \mathrm{q}=$ investigacion + educativa $\% 2 \mathrm{c}+\mathrm{ev}$ aluacion $+y+$ pedagogías + crit icas.

Ribas, S. (2006). La regulación del proceso de composición escrita en grupo: análisis de la utilización de pautas de revisión. En Camps A. y Ballesteros C. Comps. El aula como espacio de investigación y reflexión: investigaciones en didáctica de la lengua (pp. 51-62). Barcelona: Gra. 